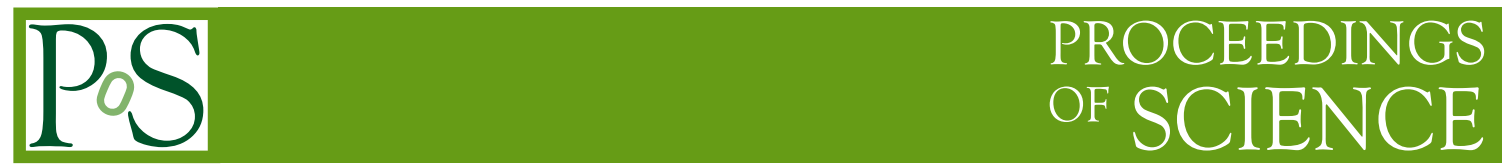

\title{
Search for resonant diboson production at the LHC
}

\author{
Matthias U. Mozer* \\ KIT \\ E-mail: Matthias.Mozer@kit.edu
}

The study of electroweak boson pair production is a powerful test of the spontaneously broken gauge symmetry of the Standard Model (SM) and can be used to search for phenomena beyond the SM. Heavy particles decaying to gauge boson pairs are predicted by many scenarios of new physics, including Extra Dimensions, and Technicolor models. We present generic searches for a heavy particle decaying to a pair of boson. Results from the ATLAS and CMS collaboration at the LHC are presented.

XXII. International Workshop on Deep-Inelastic Scattering and Related Subjects, 28 April - 2 May 2014

Warsaw, Poland

${ }^{*}$ Speaker. 


\section{Introduction}

The standard model (SM) of particle physics has been very successful in describing the highenergy physics phenomena investigated so far. One of the predictions of the SM is the existence of a scalar particle, known as the Higgs boson, associated with the spontaneous breaking of the electroweak (EW) symmetry and responsible for the masses of the SM particles. The recent discovery by the ATLAS and CMS Collaborations of a particle compatible with the SM predictions for the Higgs boson provides further verification of the SM [1,2]. In view of large loop corrections to the Higgs boson mass, the question arises whether the measured Higgs boson mass is the result of fine-tuned constants of nature within the SM or whether new physics at the $\mathrm{TeV}$ scale stabilizes the Higgs field vacuum. This question can be reformulated in terms of the large difference between the mass of the Higgs boson and the Planck scale $M_{\mathrm{Pl}}$, where the gravitational force is expected to have the same strength as the other fundamental forces $\left(M_{\mathrm{Pl}} \sim 10^{16} \mathrm{TeV}\right)$.

In many theoretical extensions of the SM, the spontaneous breaking of the EW symmetry is associated with new strong dynamics appearing at the $\mathrm{TeV}$ scale. For instance, the origin of the new dynamics may be due to new interactions [3] or a composite Higgs boson [4]. These extensions of the SM predict the existence of new resonances coupling to pairs of massive vector bosons (VV, where $\mathrm{V}=\mathrm{W}$ or $\mathrm{Z}$ ).

Models extending the number of spatial dimensions are of particular interest in the attempt to explain the apparently large difference between the EW and the gravitational scale. Some of these models predict the existence of a so-called tower of Kaluza-Klein (KK) excitations of a spin-2 boson, the KK graviton. The WW and ZZ channels are some of the possible decay modes of the Randall-Sundrum (RS) graviton [5] in warped extra dimension models. The original RS model (here denoted as RS1) can be extended to the bulk graviton model, which addresses the flavor structure of the SM through localization of fermions in the warped extra dimension [6].

\section{Leptonic Final States}

The search for di-boson resonances in all leptonic final states has the great advantage, that the signature of multiple leptons, possibly in combination with missing transverse energy (MET), is rare in processes with high production cross section, so that little background is expected. However, the branching ratios of vector bosons to leptons are quite small, so that the signal efficiency suffers correspondingly. This issue is exacerbated by the fact that all studies presented here rely on electrons and muons only. Depending on the number of neutrinos in the final state, the mass resolution for the candidate resonance ranges from excellent $(\mathrm{ZZ} \rightarrow 4 \ell)$ via reasonable $(\mathrm{WZ} \rightarrow 3 \ell v$, the mass being reconstructed with the $\mathrm{W}$ mass constraint) to poor (WW $\rightarrow 2 \ell 2 v$ ).

The CMS [7] and ATLAS [8] experiments both have analyzed approximately $20 \mathrm{fb}^{-1}$ of LHC data, taken at a center-of-mass energy of $8 \mathrm{TeV}$, in search of a resonance decaying to the WZ final state $[9,10]$. Both analysis proceed quite similarly: A pair of same flavor, opposite charge leptons compatible with the invariant mass of the $\mathrm{Z}$ is selected. Another lepton is required and interpreted as originating from the $\mathrm{W}$ decay. The invariant mass of the hypothetical resonance is reconstructed using the the kinematic quantities of the three leptons combined with the missing transverse energy (MET) under the constraint of the known $\mathrm{W}$ mass. The final spectra of reconstructed mass are 
dominated by SM WZ production, which steeply falls as function of the mass (see Fig. 1). As a result, both experiments can put stringent limits on the existence of possible WZ resonance, for example a second, heavy W boson, which is excluded below a mass of $1.45 \mathrm{TeV}$ by the CMS and below $1.52 \mathrm{TeV}$ by the ATLAS experiment at $95 \%$ confidence level (CL).
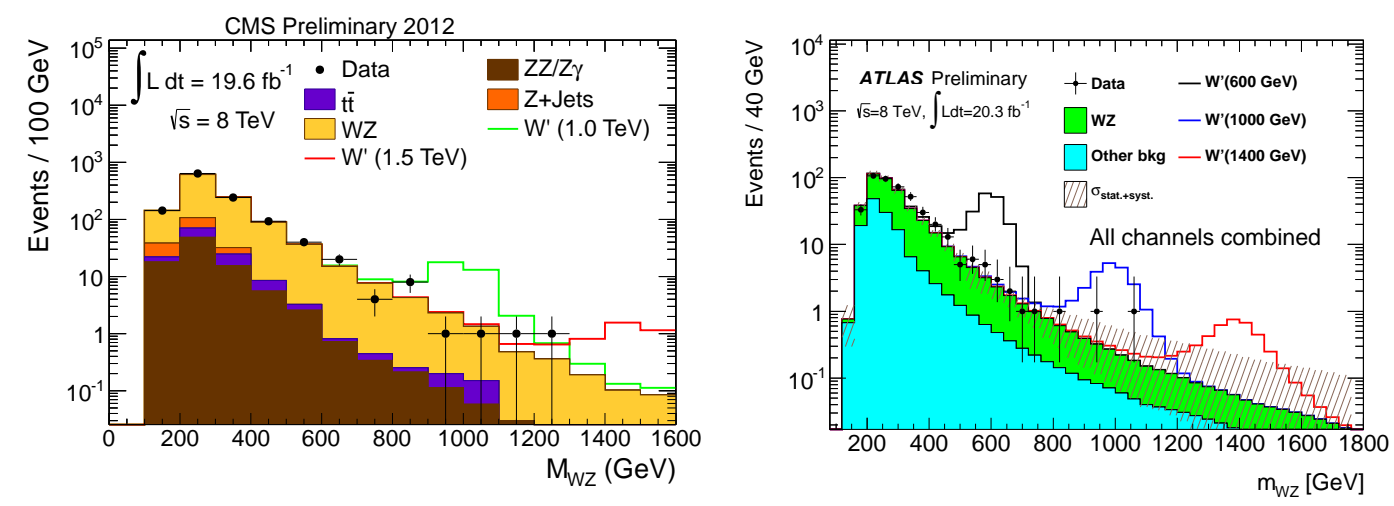

Figure 1: Invariant mass distributions for the three lepton and MET system using the $\mathrm{W}$ mass constraint in CMS (left) and ATLAS (right). Figures from Refs. [9, 10].

Additionally, the ATLAS collaboration has prepared an analysis of the entire $7 \mathrm{TeV}$ dataset of the LHC (about $5 \mathrm{fb}^{-1}$ ) in search for a WW resonance, where both W bosons decay leptonically [11]. The study is a combination of three lepton flavor combinations ee, $\mu \mu$ and e $\mu$. In the channel with same-flavor leptons, an explicit veto on the invariant mass of the $\mathrm{Z}$ boson is applied. Even with this veto in place, there is still a substantial background from the Drell-Yan process. Backgrounds from leptonically decaying top-quark pairs are suppressed by requiring the absence of jets with a b-tag. Nevertheless, top backgrounds play a dominant role in the opposite flavor channel, where the Drell-Yan background is absent. The invariant mass of the resonance cannot be reconstructed in the leptonic WW decays due to the presence of two neutrinos in the final state. Instead, the search is performed in the spectrum of the transverse mass of the di-lepton and MET, resulting in poor signal resolution. The combined result of the search in all three flavor combinations is interpreted as a limit on the presence of several different graviton hypothesis, excluding Randall-Sundrum and bulk Randall-Sundrum gravitons of masses lower than $1.23 \mathrm{TeV}$ and 0.84 $\mathrm{TeV}$ at $95 \% \mathrm{CL}$, respectively.

\section{Hadronic Final States}

Including hadronnic $\mathrm{V}$ decays in the analysis substantially increases the visible branching fraction, especially for the Z. However, single V production in association with jets ("V+jets") becomes a dominating background, as the misidentification of QCD jets as $\mathrm{V}$ decay is much more likely than the misidentification of QCD products as leptons. For moderate resonance masses, the hadronic V decay is reconstructed as a pair of jets consistent with the mass of the V. An additional complication arises for very heavy resonances, where the decay boson has very high momentum. In the case that the transverse momentum of the boson is larger than roughly twice the boson mass divided by the jet radius, most $\mathrm{V}$ decays will be reconstructed as a single jet. This is effect can 
be clearly seen in Fig. 2 (left), which shows the reconstruction efficiency of a ZZ resonance being reconstructed as combination of a leptonic $\mathrm{Z}$ decay and a jet pair, compared to the efficiency of reconstructing the resonance as combination of a leptonic $\mathrm{Z}$ and a single jet with mass compatible with the $\mathrm{Z}$ boson mass.
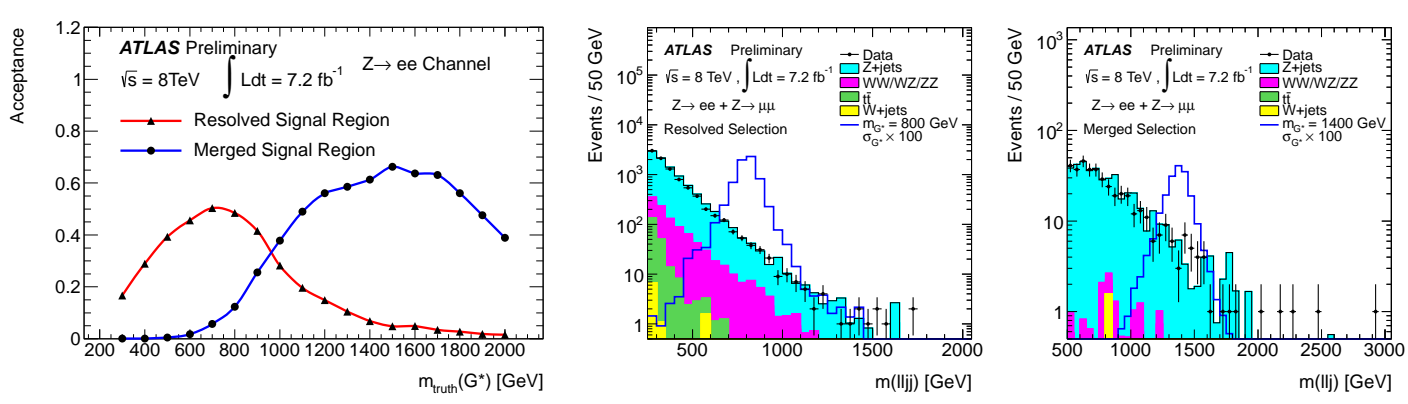

Figure 2: ATLAS results for ZZ production in the semileptonic channel. Efficiency for the di-jet and singlejet channels (left), $\ell \ell$-dijet invariant mass spectrum for the dijet channel (middle) and $\ell \ell$-jet invariant mass for the single-jet channel (right). Figures from Ref. [12]

The ATLAS collaboration used two separate analysis channels, one with dijets and one with single massive jets to search for $\mathrm{ZZ}$ resonances in the complete $7 \mathrm{TeV}$ dataset [12]. The search is performed in the semi-leptonic channel. The leptonic $\mathrm{Z}$ is reconstructed from a pair of same flavor, opposite sign leptons compatible with the $\mathrm{Z}$ mass. Additionally jet pairs or single jets compatible with the $\mathrm{Z}$ mass are selected. As all decay products of the hypothetical resonance are reconstructed in the detector, its invariant mass can be easily reconstructed. The final mass resolution is limited by uncertainties in the jet calibration. The invariant mass spectra of the dijet (single jet) channel can bee seen in Fig. 2 middle (right), respectively, showing that the observed data are compatible with the large Z+jets background. Limits are extracted on several graviton models, leading for example to a lower limit of $850 \mathrm{GeV}$ at $95 \% \mathrm{CL}$ on the mass of a bulk Randall-Sundrum graviton.

Beyond the jet mass, the distribution of the constituents within a jet can provide further separation between jets originating from $\mathrm{V}$ decays and quark or gluon hadronization. Two methods are widely used in the CMS collaboration at the moment to exploit this: jet-pruning and N-subjettiness. The jet-pruning algorithm refines the reconstruction of the jet-mass by repeating the sequential combinations of the initial jet reconstruction, removing combinations that are soft or at large angles [13]. This improves the the performance of the requirement that the jet mass be compatible with the $\mathrm{Z}$ mass by improving the mass resolution of hadronic $\mathrm{V}$ decays as well as reducing the number of quark and gluon jets that are reconstructed with high jet masses. For the $\mathrm{N}$-subjettiness we define a measure, $\tau_{N}$, of the compatibility of a jet having $N$ subjets. The value of $\tau_{N}$ tends to zero as a jet becomes more consistent with $\mathrm{N}$ subjets. For discriminating merged $\mathrm{V}$ jets with two subjets and QCD jets with a single subjet the ratio of $\tau_{21}=\tau_{2} / \tau_{1}$ has been found to be especially effective [13].

In simulation, the jet sub-structure is influenced by the phenomenological description of parton shower and hadronization. To ensure that the variables are reasonably described in simulation and possible shortcomings corrected, it is important to validate the methods on data. This is feasible for $\mathrm{W}$ decays, where semi-leptonically decaying top quark pairs provide a natural proving ground 
for the study of hadronic W decays. A single lepton, together with a b-tagged jet and MET are required to ensure the presence of a top quark. On the opposite site of the event, there is with high likelihood a $\mathrm{W}$ boson, which can be used to study the jet sub-structure variables with a tagand-probe approach [13], showing that modern simulations can reproduce the internal structure of hadronic W decays remarkably well.

The CMS collaboration uses these jet substructure discriminant in a coordinate suite of searches: the semi-leptonic WW and ZZ channel [14] as well as an all-hadronic search, which is sensitive to $\mathrm{WW}, \mathrm{ZZ}$ and $\mathrm{WZ}$ final states as well as new resonances decaying to an ordinary jet in addition to a hadronically decaying $\mathrm{W}$ or $\mathrm{Z}$ (such as excited quarks) [15]. The reconstruction of the leptonic $\mathrm{V}$ follows is similar to the fully leptonic analysis described above, while the hadronic $\mathrm{V}$ is identified by the jet substructure variables discussed in the previous section, substantially suppressing background from $\mathrm{V}+$ jets (dijets) in the semileptonic (fully hadronic) analysis, respectively. Fig. 3, shows the resulting mass spectra in the three final states.
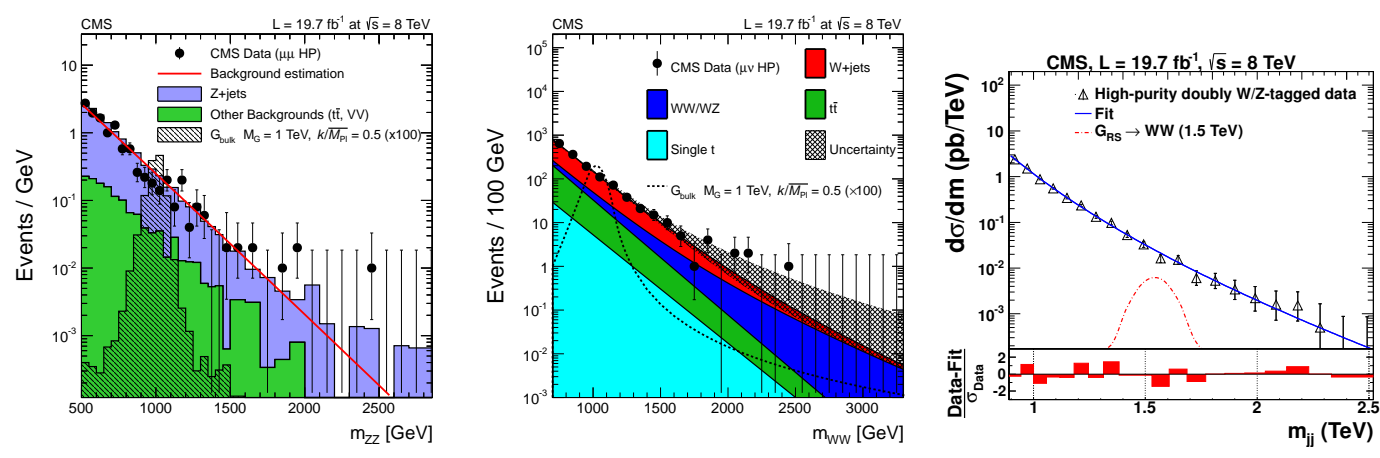

Figure 3: Hadronic resonance searches at CMS. Left: invariant mass spectrum for the di-lepton and jet system in the ZZ resonance search. Middle: Invariant mass spectrum of the lepton, jet and MET system, using the W-mass constraint, in the WW decay channel. Right: the invariant mass spectrum for two V-tagged jets. Figures from Refs. [14, 15].

The limits of these studies are interpreted as limits on a bulk graviton model, for each channel and in the combination, following a similar procedure as the combination of Higgs search channels [1] (See Fig. 4). Limits for the semi-leptonic analysis are additionally derived in a modelindependent manner as function of the resonance width and mass. These limits are accompanied by and efficiency parametrization that allows the comparison of arbitrary models with these limits.

In the all hadronic final state another resonant diboson process becomes accessible: the search for new resonances decaying to two SM Higgs bosons. As the SM Higgs boson has a large branching fraction to b-quarks, background can be effectively suppressed by the application of suitable b-jet identification criteria. This has been exploited by the ATLAS collaboration in an analysis for a resonance decaying to two Higgs bosons in the four-jet final state [16]. To suppress the large background from QCD multijet events, the four b-tagged jets are required to form back-to-back pairs with high transverse momentum, each pair being compatible with the Higgs boson mass. The background spectrum is controlled from sidebands in the dijet mass as well as samples with loser b-tag requirements. Fig. 5 (left) shows the four jet invariant mass. The background is dominated by multijet events, which carries considerable systematic uncertainties due to the quadruple b-tag 

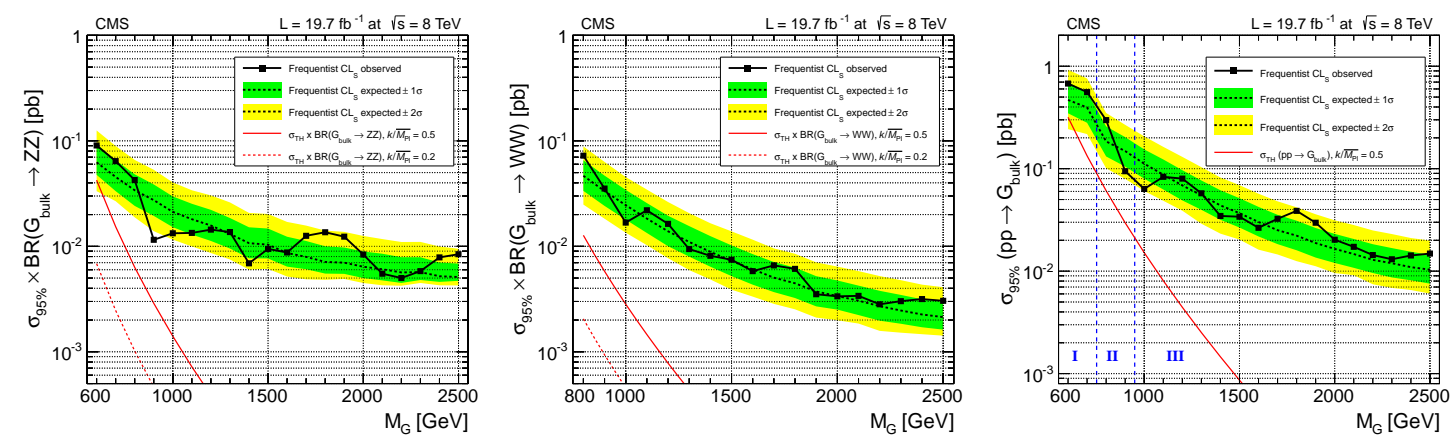

Figure 4: Limits on a Bulk Graviton model using CMS semi-leptonic and fully hadronic data. Left: limit in the ZZ semi-leptonic channel. Middle: Limit int the WW semi-leptonic channel. Right: Combination of the WW and ZZ semi-leptonic channels with the all hardonic channel. Figures from Ref. [14]

requirement. The observed mass spectrum is compared to expectations, and limits on a hypothetical graviton are extracted (see Fig. 5). At very high masses, the analysis loses power to the jet merging effect described above. The possibility of b-tagging in merged jet topologies is under active investigation in CMS and ATLAS, but no corresponding di-boson analysis has been published yet.
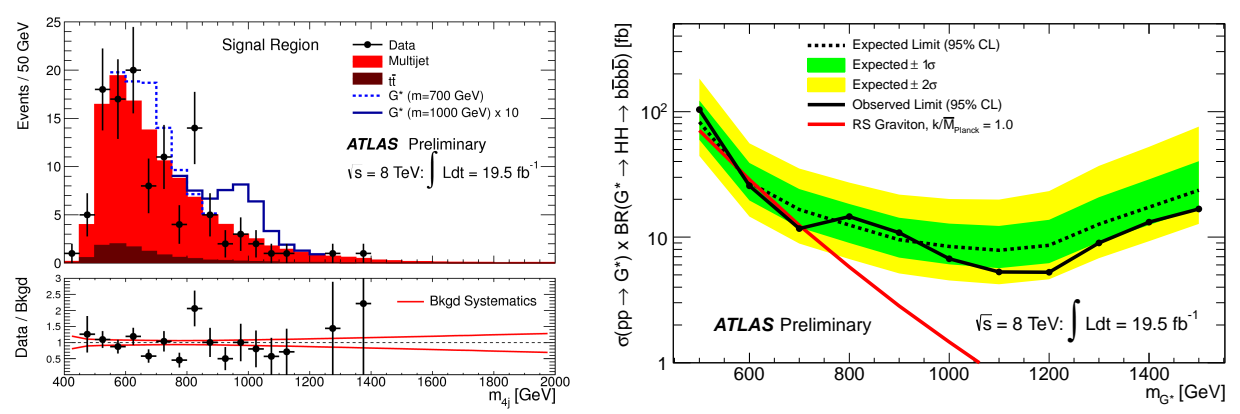

Figure 5: Search for a resonance decaying to two Higgs bosons with the ATLAS experiment. Left: Invariant mass spectrum of the four b-tagged decay jets. Right: The corresponding limit on a bulk Randall-Sundrum graviton. Figures from Ref. [16].

\section{Conclusion and Outlook}

The first run of the LHC has produced a plethora of searches for di-boson resonances in a variety of final states, all with negative results. The corresponding limits push the possible masses of gravitons and similar resonances into the $\mathrm{TeV}$ range, far beyond previous results from the Tevatron. The second run of the LHC promises even stricter limits, or possibly a discovery, largely due to the increased beam energy, which increases the parton luminosity disproportionally at high energies of the parton center of mass system. With the heavier obtainable resonances, the decay boson transverse momenta will be even higher and jet-substructure techniques will become even more important. 


\section{References}

[1] S. Chatrchyan et al. [CMS Collaboration], "Observation of a new boson with mass near $125 \mathrm{GeV}$ in pp collisions at $\sqrt{s}=7$ and 8 TeV,” JHEP 1306 (2013) 081 [arXiv:1303.4571 [hep-ex]].

[2] G. Aad et al. [ATLAS Collaboration], "Observation of a new particle in the search for the Standard Model Higgs boson with the ATLAS detector at the LHC," Phys. Lett. B 716 (2012) 1 [arXiv:1207.7214 [hep-ex]].

[3] L. Susskind, "Dynamics of Spontaneous Symmetry Breaking in the Weinberg-Salam Theory," Phys. Rev. D 20 (1979) 2619.

[4] G. F. Giudice, C. Grojean, A. Pomarol and R. Rattazzi, “The Strongly-Interacting Light Higgs,” JHEP 0706 (2007) 045 [hep-ph/0703164].

[5] L. Randall and R. Sundrum, “A Large mass hierarchy from a small extra dimension,” Phys. Rev. Lett. 83 (1999) 3370 [hep-ph/9905221].

[6] K. Agashe, H. Davoudiasl, G. Perez and A. Soni, "Warped Gravitons at the LHC and Beyond," Phys. Rev. D 76 (2007) 036006 [hep-ph/0701186].

[7] S. Chatrchyan et al. [CMS Collaboration], "The CMS experiment at the CERN LHC," JINST 3 (2008) S08004.

[8] G. Aad et al. [ATLAS Collaboration], "The ATLAS Experiment at the CERN Large Hadron Collider," JINST 3 (2008) S08003.

[9] The CMS Collaboration, "Search for W'/technirho in WZ using leptonic final states," CMS-PAS-EXO-12-025.

[10] The ATLAS collaboration, "Search for a $W Z$ resonance in the fully leptonic channel using $p p$ collisions at $\sqrt{s}=8 \mathrm{TeV}$ with the ATLAS detector," ATLAS-CONF-2014-015.

[11] G. Aad et al. [ATLAS Collaboration], "Search for new phenomena in the $W W$ to $\ell v \ell$ ' $v$ ' final state in $p p$ collisions at $\sqrt{s}=7 \mathrm{TeV}$ with the ATLAS detector," Phys. Lett. B 718 (2013) 860 [arXiv:1208.2880 [hep-ex]].

[12] The ATLAS Collaboration, "Search for resonant ZZ production in the ZZ -> llqq channel with the ATLAS detector using $7.2 \mathrm{fb}-1$ of sqrt(s) = $8 \mathrm{TeV}$ pp collision data," ATLAS-CONF-2012-150.

[13] The CMS Collaboration, "Identifying Hadronically Decaying Vector Bosons Merged into a Single Jet,” CMS-PAS-JME-13-006.

[14] V. Khachatryan et al. [CMS Collaboration], "Search for massive resonances decaying into pairs of boosted bosons in semi-leptonic final states at sqrt(s) = $8 \mathrm{TeV}$," [arXiv:1405.3447 [hep-ex]].

[15] V. Khachatryan et al. [CMS Collaboration], "Search for massive resonances in dijet systems containing jets tagged as $\mathrm{W}$ or Z boson decays in pp collisions at sqrt(s)= $8 \mathrm{TeV}$," [arXiv:1405.1994 [hep-ex]].

[16] The ATLAS collaboration, "A search for resonant Higgs-pair production in the $b \bar{b} b \bar{b}$ final state in $p p$ collisions at $\sqrt{s}=8 \mathrm{TeV}$," ATLAS-CONF-2014-005. 